\title{
Angiogenesis inhibitors in the treatment of breast cancer: exploring avenues of new therapeutic targets
}

\begin{abstract}
Breast cancer is a debilitating disease, which, according to the National Breast Cancer Foundation will affect one in eight women. While the etiology of the disease is multi factorial, angiogenesis, the growth of new vessels from pre-existing vessels, plays a central role in the progression of the disease. Over the past few decades, research has shown that angiogenesis may be the fundamental step that transitions benign tumors into malignant tumors. Endothelial cells (ECs) secrete pro-angiogenic factors that facilitate vascular network formation, thus providing tumors with the necessary oxygen and nutrients for growth and metastasis. Angiogenesis inhibitors aim to interfere with the signaling pathways that promote tumor angiogenesis. Angiogenic inhibition may be accomplished via targeting of pro-angiogenic molecules, cell surface receptors, signaling pathways, or through inhibition of other molecular events. In addition to these emerging therapies, there are several anti-angiogenic drugs that have been proven to be clinically useful in the treatment of breast cancer. It is anticipated that research with these agents will prevent the expansion of tumor masses, cause tumor dormancy, or sustain regression of the tumor. This review aims to examine some of the most recent emerging anti-angiogenic therapies along with present clinically available anti-angiogenic drugs in the treatment of breast cancer.
\end{abstract}

Volume 2 Issue 6 - 2015

\author{
Ayesha Patel,Abigail Hielscher \\ Department of Biomedical Sciences, Georgia-Philadelphia \\ College of Osteopathic Medicine, USA
}

Correspondence: Abigail Hielscher, Department of Biomedical Sciences \#133, Georgia-Philadelphia College of Osteopathic Medicine, Suwanee, GA 30024, USA, Tel (678) 225-7463, Fax (678) 225-7469, Email abigailhi@pcom.edu

Received: April 27, 20I5 | Published: June 24, 2015

\section{Introduction}

Angiogenesis is the physiological process through which new blood vessels form from pre-existing blood vessels and it is a critical part of development and wound healing. ${ }^{1}$ This vascular network can originate in three ways: new blood vessels can sprout from existing vessels; new vessels can arise from bone marrow derived endothelial progenitor cells; or capillary walls can invaginate into their lumen and cause existing vessels to split into two new vessels. ${ }^{1}$ In order for any of these processes to occur, there must be a balance between proangiogenic and anti-angiogenic growth factors, as any tip in balance can lead to physiological problems. ${ }^{2}$ Of these factors, vascular endothelial growth factor (VEGF), which regulates endothelial cell (EC) proliferation, permeability and survival, is one of the most specific and critical regulators of angiogenesis., ${ }^{2,3}$ Loss of even a single VEGF allele during embryogenesis can result in the death of the embryo, which implicates how essential this growth factor is in early development. ${ }^{3}$ In addition to stimulatory molecules, other intrinsic factors such as, hypoxia, hypoglycemia, and mechanical stress can also induce angiogenesis as well. ${ }^{2}$

Over the past few decades, research has shown that angiogenesis may be the fundamental step that transitions benign tumors into malignant tumors. ${ }^{1}$ Tumors require a rich vascular supply, which provides oxygen and nutrients necessary for growth. ${ }^{4}$ Tumor angiogenesis is unique in that the vascular structures grow in an unregulated and abnormal fashion. ${ }^{1}$ The imbalance of pro- and antiangiogenic signaling within tumors creates an abnormal vascular network characterized by dilated and hyper permeable vessels. ${ }^{1}$ Furthermore, this atypical vasculature enables the tumor cells to become more resistant to hypoxia, which helps them overcome the cytotoxic functions of immune cells and evade the host immune system. ${ }^{1}$

According to the National Breast Cancer Foundation, breast cancer is the second leading cause of death and one out of every eight women will be diagnosed with breast cancer in their lifetime. Breast cancer patients generally have one of the three types of tumors: estrogen and/or progesterone receptor positive (e.g. hormone positive (HR) tumors), human epidermal receptor 2 amplified (HER2-amplified) tumors, or triple negative breast cancer (TNBC) tumors. ${ }^{5}$ Since 2008, there has been a $20 \%$ increase in breast cancer cases. ${ }^{4}$ Along with most other cancers, its etiology can be due to multi factorial reasons and as such, is difficult to pinpoint the optimum form of treatment. The primary treatment for breast cancer is complete removal of the tumor tissue, either through mastectomy or lumpectomy. ${ }^{4}$ If this treatment is not an option, adjuvant therapies such as radiation, chemotherapy, anti-hormone therapy, targeted HER2 therapy and anti-angiogenesis therapies can then be administered. ${ }^{4}$

Targeted cancer therapy involves understanding the specific characteristics of cancer cells that make them different from normal cells. By targeting these specific factors, scientists can focus on eradicating cancerous cells while leaving healthy cells unharmed, thus lessening the likelihood of side effects during cancer treatment. In 1971 , Folkman $\mathrm{J}^{6}$ proposed that targeting pro-angiogenic factors could be useful in cancer treatment. ${ }^{6}$ Given the extent of neo vessel formation in breast tumors, angiogenesis inhibitors are a widely investigated and utilized form of chemotherapy. Angiogenesis inhibitors aim to interfere with the signaling pathways that allow molecules to bind to endothelial cell (EC) receptors and facilitate blood vessel growth, thus slowing or inhibiting tumor growth and metastasis. ${ }^{3}$

Currently, there are over 20 anti-angiogenic drugs that have proven to be clinically useful in the treatment of breast cancer. Although these drugs will not likely cure breast cancer, it is anticipated that research with these agents will prevent the expansion of tumor masses, cause tumor dormancy, or sustain regression of the tumor. Because there are number of ways vessels can be targeted, this review aims to examine some of the most recent emerging anti-angiogenic therapies along with present clinically useful drugs in the treatment of breast cancer. 


\section{Clinically available anti-angiogenic therapies}

Tamoxifen is a partial estrogen receptor antagonist and has proven to be an effective therapeutic agent in the treatment of estrogen receptor positive breast cancers. ${ }^{7}$ A review by Jordan $\mathrm{C}^{7}$ stated that estrogen, upon binding to its receptor, causes an increase in TGF- $\alpha$, a transforming growth factor which aids tumor cell survival by promoting tumor angiogenesis. ${ }^{7}$ By administering tamoxifen, the amount of estrogen bound to estrogen receptors decreases, which in turn, decreases the concentration of TGF- $\alpha$ and thus angiogenesis. ${ }^{7}$

In order to better understand a mechanism(s) responsible for tamoxifen's anti angiogenic effects, Haran EF et al., ${ }^{8}$ conducted an experiment to investigate the effects of tamoxifen on the growth, distribution and density of ECs in tumors from estrogen supplemented or non-supplemented xenograft MCF7 breast tumors. ${ }^{8}$ After 2 weeks of treatment, their results demonstrated that the average tumor size in the control tumors (non tamoxifen injected cells) was $1.64 \mathrm{~cm} 3(\mathrm{P}<0.007)$ while the average tumor size in the tamoxifen-injected tumor cells was $0.67 \mathrm{~cm} 3(\mathrm{P}<0.03) .{ }^{8}$ Investigating EC density, the authors showed that the percent area occupied by ECs, identified through use of biotinylated lectin antibody, was $10.2 \%$ in control tumors and $4.4 \%$ in tamoxifen injected tumor cells $(\mathrm{P}<0.0001) .{ }^{8}$ Overall, it appears that tamoxifen reduced EC proliferation, which in turn halted tumor growth. ${ }^{8}$ Results of this study suggest that tamoxifen alone can be used as a form of anti-angiogenic treatment for breast cancer. ${ }^{7,8}$

Given the role for tamoxifen as an anti-angiogenic agent, Gagliardi A \& Collins $\mathrm{DC}^{9}$ sought to examine the anti-angiogenic properties of tamoxifen in combination with other estrogen receptors antagonist drugs. ${ }^{9}$ The authors hypothesized that partial estrogen antagonists, clomiphene, tamoxifen, and nafoxidine if combined synergistically with 17- $\beta$ estradiol or heparin, would inhibit angiogenesis in the chick egg chorioallantoic membrane (CAM), a model for investigating neovascularization. ${ }^{9}$ They found that combining each estrogen receptor antagonist individually with either 17- $\beta$ estradiol or heparin decreased angiogenesis in the CAM model in a dose dependent manner. ${ }^{9}$ It can be inferred that they used the anticoagulant, heparin, in order to allow the body's natural clot apoptotic mechanisms to work efficiently, and $17-\beta$ estradiol, a hormone essential in the maintenance of the female reproductive system, was added to stabilize the tissue. For example, when clomiphene was administered alone, it inhibited angiogenesis by $23 \%$ but when given in combination with $17 \beta$-estradiol or heparin, angiogenesis was inhibited by $37 \%$ and $29 \%$ respectively. ${ }^{9}$ When tamoxifen was given alone, angiogenesis was inhibited by $10 \%$ but when combined with $17 \beta$-estradiol or heparin, angiogenesis was inhibited by $37 \%$ and $20 \%$ respectively, ${ }^{9}$ Nafoxidine gave similar results: $10 \%$ when given alone and $14 \%$ with $17 \beta$-estradiol and $15 \%$ with heparin. ${ }^{9}$ Similar to previous studies, it was proposed that tamoxifen reduced angiogenesis as a result of interfering with estrogen binding to its receptor. ${ }^{9}$ As a result, it can be concluded that, when combined with $17 \beta$-estradiol or heparin, partial estrogen antagonists exert greater anti-angiogenesis activity. This therapeutic strategy may be a useful treatment modality for breast cancer.

Growing evidence demonstrates that vascular endothelial growth factor (VEGF), a growth factor that facilitates EC proliferation, is a critical mediator of angiogenesis. Ferrara $\mathrm{N}$ et al. ${ }^{10}$ reported the first suppression of EC growth in vivo using anti-antibodies directed against VEGF. Here, the authors subcutaneously injected anti-VEGF monoclonal antibodies in nude mice harboring one of three different tumor cell lines: the G55 glioblastoma multiforme, the SK-LMS-1 leiomyosarcoma and the A673 rhabdomyo-sarcoma. ${ }^{10}$ Results demonstrated that tumor growth inhibition ranged between 70\%-95\% in in vivo trials whereas the antibodies had no effects on the tumor cell lines in vitro. ${ }^{10}$ These results provided the first demonstration that anti-VEGF therapy could be useful in treating tumor tissue in vivo. ${ }^{10}$

Later in 2003, bevacizumab, a monoclonal antibody that targets VEGF, was first investigated in a phase 1-2 clinical trial in women with chemotherapy refractory metastatic breast cancer. ${ }^{11}$ The mechanism of bevacizumab is that it prevents VEGF from binding with its receptor, VEGFR2. ${ }^{11}$ In this study, patients $(n=75)$ received increasing doses of bevacizumab $\left(3,10\right.$, or $20 \mathrm{mg} / \mathrm{kg}$ ) every two weeks. ${ }^{11}$ After 155 days, $16 \%$ of patients had stable disease or a confirmed continued response. ${ }^{11}$ The remarkable extended period of stable disease with minimum side effects allowed the drug to continue to the next phases of clinical trials. ${ }^{11}$ By 2005, Miller KD et al. ${ }^{12}$ had reported the first completed phase III trial of antiangiogenic therapy in patients with metastatic breast cancer. ${ }^{12}$ Their study aimed to compare the efficacy and safety of a common chemotherapeutic agent, capecitabine, alone or in combination with bevacizumab in patients who had previously been treated with anthracycline and a taxane. ${ }^{12}$ Patients were given capecitabine $(2500 \mathrm{mg} / \mathrm{m} 2 / \mathrm{d})$ twice a day every three weeks, alone or in combination with bevacizumab $(15 \mathrm{mg} / \mathrm{kg}) .{ }^{12}$ Their results showed that combination therapy significantly increased response rates $(19.8 \%$ vs. $9.1 \%, \mathrm{P}=0.001)$ but not progression free survival. ${ }^{12}$ It was also reported that bevacizumab associated toxicities resulted in hypertension and proteinuria in approximately $18 \%$ of the patients. ${ }^{12}$ Because their results did not show a therapeutic advantage, the authors concluded that additional pro-angiogenic factors aside from VEGF might be responsible for their observations. ${ }^{12}$ The results of this study suggest that the optimal time to intervene in tumor angiogenesis is important and the effects of bevacizumab might not be as effective when used in combination with other chemotherapeutic agents. ${ }^{12}$

To further investigate the properties of bevacizumab in combination with other chemotherapeutic drugs, Miller KD et al. ${ }^{12}$ conducted the next randomized phase III clinical trial to study the safety and efficacy of using paclitaxel, a mitotic inhibitor, alone or in combination with bevacizumab in patients with metastatic breast cancer. ${ }^{12}$ The researchers randomly assigned 772 patients into two groups: half received paclitaxel and the other half received paclitaxel plus bevacizumab. ${ }^{12}$ Results of the study showed that patients who received paclitaxel plus bevacizumab had significantly prolonged progression free survival but not overall survival. ${ }^{12}$ According to the results, the authors believe that bevacizumab targeted the VEGF-A isoform, which is a more potent factor in inducing vasodilation and pathogenic angiogenesis. They also found that treatment with bevacizumab in initial of the experiment were more effective when angiogenic pathways were more VEGF- dependent. ${ }^{13}$ Later in the trial, it was found that patients were developing resistance to bevacizumab over time, which may account for the reason that the drug is ineffective in later parts of treatment. ${ }^{12}$ Thus, the results of this study suggest that the most successful clinical application of angiogenesis inhibitors is during the initial periods of cancer treatment when there are less angiogenic pathways present. ${ }^{12}$

This overall history of bevacizumab is important to understand because it was one of the first monoclonal antibody therapies used to directly target angiogenesis. After the randomized phase III clinical trial by Miller KD et al., ${ }^{12}$ bevacizumab was granted an accelerated approval by the US Food and Drug Administration (FDA). ${ }^{14}$ The FDA reversed this decision two years later in 2010 because there were safety concerns that the risks outweighed the benefits in terms of prolonged progression free survival. ${ }^{14}$ Although bevacizumab is no longer a treatment modality for breast cancer, the drug however is approved for the treatment of many other types of cancer such as metastatic 
colorectal cancer, metastatic renal cell carcinoma, metastatic cervical cancer, and ovarian cancer when combined with chemotherapy. ${ }^{14}$

Although anti-angiogenic agents tested in clinical trials have proven beneficial, the clinical utility of these studies remains uncertain due to the absence of data on overall survival, development of additional toxicities, and substantial costs associated with the ongoing research. ${ }^{15}$ Studies have also suggested that the population of patients selected for research is essential since some metastatic breast cancer patient's benefit from anti-angiogenic agents over others. ${ }^{15}$ The observation that anti-angiogenic agents impart resistance to the drug over time is an additional challenge for conducting long-term studies. ${ }^{15}$ Furthermore, since there are numerous pro-angiogenic factors that regulate tumor growth and angiogenesis, targeting one or two factors may not be a beneficial form of long-term treatment. ${ }^{15}$ With regard to targeting angiogenic pathways, research has still not pinpointed exact biological predictors of clinical success; hence additional work on anti-angiogenic factors necessitate further investigation so as to elucidate better targeted therapies.

\section{Emerging anti-angiogenic therapies}

In recent years, many studies have utilized in vitro and in vivo models to investigate novel approaches to target angiogenesis. This section will examine some of the most recent emerging antiangiogenic therapies in targeting angiogenic mechanisms in breast cancer.

\section{Inhibition through direct targeting of pro-angiogenic molecules}

As previously discussed, one way to target angiogenesis is to directly target pro-angiogenic molecules, thus preventing association with their cognate receptors. Angiopoietin-2 (Ang2) is one of several pro-angiogenic factors that play a critical role in tumor progression. ${ }^{16}$ Recently, He T et al., ${ }^{16}$ utilized miRNA-542-3p to target Ang2 and thus tumor angiogenesis. ${ }^{16}$ In this study, the authors administered miRNA-542-3p to tumor bearing mice and observed that miRNA542-3p inhibited translation of Ang2 mRNA by binding to its 3'UTR and as a result, reduced EC proliferation and tumor angiogenesis. ${ }^{16}$ To investigate anti-angiogenic properties of miRNA-542-3p in-vivo, they used lenti viruses containing miRNA-542-3p for treatment of tumor bearing mice. ${ }^{16}$ The results demonstrated that LV-miRNA-542-3p strongly suppressed tumor volume and also relative vessel density in vivo. ${ }^{16}$ To observe anti-angiogenic properties in vitro, a tube formation and spheroid- based EC sprouting assay showed that miRNA-542-3p suppressed capillary tube formation and cell-sprouting ability from human umbilical vein endothelial cells (HUVECs). ${ }^{16}$ In previous work, it was shown thatAng2recruited TIE2-expressing monocytes (TEMs) that facilitated angiogenesis in mouse tumor models including spontaneous MMTV-PyMT mammary and RIP1-Tag2 pancreatic islet adenocarcinomas. ${ }^{17}$ As a result, the authors additionally proposed that miRNA-542-3p targeted TEM signaling through its actions on Ang2, which further decreased tumor metastatic and angiogenic properties. ${ }^{16}$ Since this is a fairly new approach to targeted therapy, the results of this experiment suggest that utilizing miRNA is a potential antiangiogenic therapeutic approach in the treatment of both breast cancer and other solid tumor malignancies.

Fibroblast growth factor (FGF) is another pro-angiogenic growth factor and signaling with its receptor (FGFR) has been linked to inflammation, cancer, and resistance to VEGF inhibitor treatment. ${ }^{18}$ Bono F et al. ${ }^{19}$ reported that the chemical SSR128129E (SSR) inhibited FGFR signaling by binding to the extracellular FGFR domain. ${ }^{19}$ To study their effects in vitro, they used HUVECs which express FGFR1, FGFR2 and FGFR4 and observed that SSR inhibited FGF-2 induced EC proliferation, migration and lamellipodia formation in a dose dependent manner. ${ }^{19}$ Furthermore, they also analyzed SSR in tumor angiogenesis, growth and metastasis using orthotopic tumor models and human xenograft tumor models. ${ }^{19}$ Here, they demonstrated that oral delivery of SSR in murine 4T1 breast tumors reduced tumor size and weight by $53 \%$ and $40 \%$ respectively. ${ }^{19}$ Tumor cells in this model also over expressed several FGFRs, while many FGF ligands were also detectable. ${ }^{19}$ Furthermore, they observed that SSR enhanced the antitumor activity of the anti-VEGFR2 antibody DC101 ( $\alpha$ VEGFR2) in pancreatic tumors as well. ${ }^{19}$ The results of this study suggest that targeting FGFR and thus limiting FGF signaling may serve as a potential anti-angiogenic therapy in cancer treatment.

\section{Inhibition through receptor targeting and signaling pathways}

Another mechanism for targeting angiogenesis is to block angiogenic receptors and signaling pathways. In 2010, Lin L et al., ${ }^{20}$ developed two small STAT3 inhibitors, FLLL31 and FLLL32, which are derived from curcumin, a bioactive substance that inhibits oncogenic events. ${ }^{20}$ To determine whether these compounds effected STAT3 target genes, including VEGF, they treated MDA-MB-231 breast cancer cells with FLLL31 and FLLL32 and examined changes in protein expression using western blot. ${ }^{20}$ It was observed that both FLLL31 and FLLL32 reduced the expression of several STAT3 target genes, including VEGF, as compared to a DMSO control and curcumin. ${ }^{20}$ The results of this study suggest that inhibition of STAT3 signaling could be a useful anti-angiogenic strategy for breast cancer as downstream target genes like VEGF are additionally inhibited. ${ }^{20}$ Although the results of this study were intriguing, in vivo studies will be necessary in order to confirm the therapeutic benefits.

Transforming growth factor (TGF- $\beta$ ) participates in tissue development, remodeling, wound healing with abnormal expression linked to various types of cancer. ${ }^{21,22}$ Wang XG et al., 22 aimed to investigate whether silencing TGF- $\beta$ would affect metastatic properties of breast cancer cells. ${ }^{22}$ In their experiment, they transfected MDA-MB-231 cells with TGF- $\beta$ siRNA and grew ECs in conditioned media from TGF- $\beta$ silenced MDA-MB-231 cells. ${ }^{22}$ Results demonstrated that knockdown of TGF- $\beta$ inhibits migratory potential of MDA-MB-231 cells through the inhibition of S100A4, a downstream target of TGF- $\beta$ signaling. Previous studies have shown that silencing of S100A4 resulted in reduced EC proliferation and angiogenesis in thyroid cancer cells as a result of down regulation of MMP-9 and VEGF. ${ }^{23}$ To directly measure angiogenic properties, network formation by human microvascular endothelial cells was found to be significantly decreased when these ECs were cultured in the presence of TGF- $\beta$ siRNA. ${ }^{22}$ The authors concluded that blocking TGF- $\beta$ reduced vascular network formation, and as a result, may be a desirable therapeutic strategy for targeting angiogenesis in tumors.

The Notch-signaling pathway is an important regulator of cell communication and mediates stem cell survival, cell fate decisions, invasion, metastasis, and self-renewal. ${ }^{2}$ In addition, research has shown that the Notch signaling pathway may be involved in angiogenesis as it has been shown to increase levels of VEFGR1 and VEGFR3. ${ }^{24}$ Funahashi $Y$ et al. ${ }^{25}$ demonstrated that VEGFR1 is a downstream target of Notch1 signaling in ECs. Their results showed that blocking the Notch signaling pathway using a protein based inhibitor resulted in decreased VEGFR-1 expression in vivo. ${ }^{25}$ The authors further showed that inhibition of Notch signaling inhibited human epidermal growth factor receptor 2 (HER2), an oncogene 
that is over expressed in many types of breast cancers. ${ }^{26}$ Several studies also suggest that there is bidirectional crosstalk between the notch receptor and the EGFR family, which consists of all HER iso forms. ${ }^{27}$ The mechanism of this communication is such that the Notch pathway maintains the signals of MAPK/P13K, which are downstream signals of HER $2 .{ }^{27}$ This cellular communication allows tumor cells to upregulate invasive capabilities. ${ }^{22}$

In 2008, Chi A et al., ${ }^{28}$ demonstrated that gamma secretase inhibitors (GSIs) are novel agents that prevent the activation of Notch signaling targets in MDA-MD-231 breast cancer cells. ${ }^{28}$ When GSIs were used in combination with radiation, there were additive cytotoxic effects in MDA-MD-231 breast cancer cells. ${ }^{28}$

Holland SJ et al., ${ }^{29}$ conducted a study to investigate whether targeting Axl, a member of the receptor tyrosine kinase (RTK) family, could be a potential form of anti-angiogenic therapy for metastatic breast cancer. ${ }^{29} \mathrm{Axl}$ activates STAT, ${ }^{29}$ previously reported to play a role in inducing angiogenesis in breast cancer. ${ }^{20}$ To determine whether disrupting Axl reduced angiogenesis in xenograft breast tumors, the authors tested the activity of R428, a small molecule inhibitor of Axl kinase.$^{29}$ They observed that R428 suppressed invasion in both MDAMB-231 and 4TI breast cancer cell lines in vitro. ${ }^{29}$ They also observed that R428 suppressed breast cancer metastasis of MDA-MB-231 cells in vivo. ${ }^{29}$ Furthermore, their results showed that R428 reduced the average number of EC vessels and VEGF - induced corneal neovascularization in vivo. ${ }^{29}$ The authors concluded that R428 most likely inhibited metastatic growth via suppression of angiogenesis. ${ }^{29}$

\section{Inhibition through external factors}

Accumulating evidence has suggested that leptin, the main adipokine secreted by adipose breast tissue, has pro-angiogenic effects. ${ }^{30}$ It has been reported that obese individuals have higher levels of leptin ${ }^{31}$ and as a result, may be more prone to developing breast cancer. Gu JW et al., ${ }^{32}$ created an animal model to observe whether postmenopausal obesity increases VEGF expression and tumor angiogenesis. In this study, the group exposed aged mice to low or high fat diets for 12 weeks. ${ }^{32}$ Murine breast cancer cells were injected subcutaneously into the fat pad and tumors were allowed to grow for a period of 4 weeks. ${ }^{32}$ At the end of the experimental period, blood samples, visceral fat and tumors were collected to measure VEGF expression. ${ }^{32}$ Their results showed that both tumor VEGF levels and plasma VEGF levels were significantly higher in mice fed a high fat diet compared to mice fed a low fat diet. ${ }^{32}$ It was also observed that postmenopausal obesity significantly increased breast tumor weight over the tumor-bearing animals fed a low fat diet. $^{32}$ To further elucidate the mechanisms of leptin's contributions to breast tumor growth, Gonzalez RR et al., ${ }^{33}$ studied the effects of leptin receptor antagonists ( $\mathrm{LPaA} 2)$ on $4 \mathrm{~T} 1$ mouse mammary tumor cells. The authors found that administeringLPaA2 prior to injecting mice with 4T1 mouse mammary tumor cells slowed the growth of mammary tumors by $90 \%$ compared to control untreated mice. ${ }^{33}$ They also found that serum VEGF/VEGFR-2 expression was much lower in mice treated with $\mathrm{LPaA} 2 .{ }^{33}$ Taken together, these results suggest that obesity may contribute to tumor angiogenesis through induction of VEGF and that inhibition of the leptin-signaling pathway may provide a new target for anti-angiogenic therapy in breast cancer.

Ellagic acid is a natural phenol antioxidant that is found in many fruits and vegetables. Wang $\mathrm{N}$ et al., ${ }^{34}$ analyzed anti-angiogenic effects of ellagic acid using in-vitro and in vivo techniques. ${ }^{34}$ Using a cell counting assay, the authors treated HUVECs with ellagic acid (ranging from 2.5 to $20 \mu \mathrm{m}$ ) in the presence and absence of VEG Fandob served that ellagic acid inhibited proliferation of HUVECs cultured in the presence of $20 \mathrm{ng} / \mathrm{ml} \mathrm{VEGF.}{ }^{34}$ A trans well assay was used to study the potential effects of ellagic acid in blocking the migration and tube formation capability of HUVECs. ${ }^{34}$ Results showed that the number of invasive HUVECs significantly decreased as the concentration of ellagic acid increased. ${ }^{34}$ A wound-healing assay further revealed that ellagic acid weakened the migratory capability of HUVECs. ${ }^{34}$ Additionally, a Matrigel assay showed that in the absence of ellagic acid, HUVECs had well-formed tubular structures while $80 \%$ of the tube network in HUVECs was destroyed following incubation with $10 \mu \mathrm{M}$ of ellagic acid. ${ }^{34}$ To test their observations in vivo, they implanted MDA-MB-231 breast cancer cells into the mammary glands of 4-week-old nude mice and treated them daily with an intra peritoneal injection of $50 \mathrm{mg} / \mathrm{kg}$ or $100 \mathrm{mg} / \mathrm{kg}$ of ellagic acid. ${ }^{34}$ The authors found that treatment with ellagic acid significantly suppressed MBA-MB-231 tumor volumes in a dose dependent manner when compared to the untreated controls. ${ }^{34}$ The mechanism proposed was that ellagic acid inhibited VEGF induced angiogenesis by directly inhibiting the phosphorylation of VEGFR-2 tyrosine kinase activity and downstream signaling pathways MAPK and PI3K/Akt in HUVECs. ${ }^{34}$ Ellagic acid also blocked angiogenesis in CAM assays evidenced from the presence of shorter vessel sprouts. ${ }^{34}$ These results suggest that natural inhibitors may serve as a future anti-angiogenic agent in breast cancer treatment and given their low toxicity, may be a desirable adjuvant to existing therapies.

\section{Inhibition through other factors}

Parsons MF et al., ${ }^{35}$ examined whether STX243, a steroidalbased drug, is more active in vivo than $2-\mathrm{MeOE} 2$, a steroidalbased compound that is a potent angiogenesis inhibitor, and a structurally similar compound, STX140. ${ }^{35}$ Although 2-MeOE2 has pro-apoptotic and anti-proliferative effects, others have shown that 2-MeOE2 exerts these effects only when administered at high concentrations. ${ }^{36-39}$ Furthermore, it is poorly bioavailable in that no detectable concentrations were found after oral administration..$^{40}$ Due to these contradictory results, Parsons MF et al.,$^{35}$ evaluated whether STX243was a more efficient anti-angiogenic agent than2-MeOE2 or the structurally similar steroidal compound STX140. ${ }^{41}$ Using an in vitro angiogenesis assay, it was found that HUVECs supplemented with VEGF and then STX243 had fewer tubules. ${ }^{35}$ In-vivo, it was observed that mice injected subcutaneously with STX243 and STX140 had significant inhibition of MDA-MB-231 tumor growth as opposed to mice injected with 2-MeOE2. ${ }^{35}$ In the in vivo Matrigel plug assay, the authors observed that in vivo angiogenesis was restricted with both STX243 and STX140 (50\% and 72\%), thus demonstrating the anti-angiogenic activity of both compounds. ${ }^{35}$ The pharmacokinetic observations of STX243 demonstrated that even after administering STX243 at different levels and also with progesterone, oral bioavailability increased almost $12 \%$, thus suggesting that this drug is orally bioavailable. ${ }^{35}$ From the results of this study, it can be concluded that STX243 impedes the growth of breast cancer tumors through anti-angiogenic mechanisms and serves as a good alternative for $2-\mathrm{MeOE} 2{ }^{35}$

Matrix metallo proteinases (MMPs) regulate angiogenesis and tumor progression by degrading the extracellular matrix (ECM) ${ }^{42}$ With regard to angiogenesis, degradation of the ECM via MMPs not only liberates pro-angiogenic growth factors, but also enables EC migration, proliferation and subsequent vessel formation. ${ }^{42}$ Contradictory to this well-established role of MMPs in angiogenesis, Bendrik C et al., ${ }^{43}$ used in-vivo breast cancer models to show that over expression of MMP-9 induced tumor regression and decreased angiogenesis. In 
their studies, adenovirus vectors carrying either MMP-9 or empty controls were injected intra-tumorally in murine breast cancer cells supplemented with estradiol and tamoxifen. ${ }^{43}$ Their results showed that AdMMP-9 increased MMP activity and significantly decreased tumor growth rate and micro vessel surface area. ${ }^{43}$ The mechanism proposed was that MMP-9 released endostatin, a $20-\mathrm{kDa} \mathrm{COOH}-$ terminal cleaved fragment from collagen XVIII,which reduced EC proliferation and migration and increased EC apoptosis. ${ }^{43}$ From the results of this study, it can be concluded that while MMPs liberate pro-angiogenic factors through degradation of the ECM, they can also induce tumor regression and decrease angiogenesis through cleavage of ECM fragments with anti-angiogenic properties. ${ }^{43}$ Results of this study imply that the balance and appropriate level of MMPs is essential in regulating cell activities.

Pasquier E et al., ${ }^{44}$ hypothesized that propranolol, a non-selective $\beta$-adrenergic receptor $(\beta-\mathrm{AR})$ antagonist commonly used to treat hypertension, could increase the efficacy of chemotherapy through its anti-angiogenic effects on ECs in breast cancer. In this study, ECs, plated atop Matrigel, were treated with different propranolol concentrations combined with other chemotherapeutic drugs such as 5-fluorouracil (5-FU) or paclitaxel..$^{44}$ The results of these studies showed that the combination of both drugs (propranolol plus 5-FU or propranolol plus paclitaxel) resulted in the greatest inhibition of angiogenesis through a significant decrease in surrounding vascular surface area but not through vascular disruption. ${ }^{44}$ Because propranolol had almost no vascular disrupting activity but did decrease vascular surface area, the mechanism proposed was due to the inhibition of new capillary tube formation rather than the disruption of pre-existing vessels. ${ }^{44}$ The results of this study implicate how the use of available drugs, such as propranolol, if combined with current chemotherapeutic agents, could provide a novel strategy for treating patients with breast cancer.

Lymphatic dissemination is an important mechanism for tumor angiogenesis as it allows the tumor to disseminate into the systemic circulation. ${ }^{45}$ Recently, Schito L et al., ${ }^{45}$ showed that hypoxiainducible factor -1 (HIF-1), a protein which is upregulated in cells growing at low oxygen concentrations, promotes lymphatic metastasis in breast cancer. ${ }^{45}$ By using mice bearing breast cancer ortho grafts, lymphangiogenesis was blocked through administration of HIF-1 inhibitors digoxin and imatibin. ${ }^{45}$ This study suggests that targeting HIF-1 may be a useful strategy to limit lymphaniogenesis and thus metastatic dissemination in breast cancer. ${ }^{45}$

\section{Discussion}

Angiogenesis is a critical part of normal of development and wound healing. ECs secrete a number of pro-angiogenic and anti-angiogenic factors and it is the balance of these factors that regulates proper vascular network formation. ${ }^{1,2}$ By creating and targeting angiogenic factors and/or their receptors in additional to signaling pathways and exogenous factors participating in angiogenesis, researchers can more effectively target tumor angiogenesis.

This review covered the anti-angiogenic efficacies and setbacks of clinically available therapies in the treatment of breast cancer and further explored the utility of new therapeutic targets which may, in the future, be used to treat breast tumor angiogenesis. With regard to currently available therapies, tamoxifen was shown to decrease the concentration of TGF- $\alpha$, an indirect regulator of angiogenesis.? Tamoxifen also reduced EC proliferation and overall EC density. ${ }^{7,8}$ Although tamoxifen successfully reduced angiogenesis when administered alone, Gagliardi A \& Collins DC $^{9}$ demonstrated that when tamoxifen was combined with $17 \beta$-estradiol or heparin, angiogenesis was inhibited by $37 \%$ and $20 \%$, respectively as opposed to $10 \%$ when used alone. ${ }^{16}$ To better target angiogenesis, Bevacizumab, a monoclonal antibody that directly targets VEGF, was developed in 2003. In its first two phases of clinical trials, Bevacizumab received a great deal of attention as it led to a remarkable extended period of stable disease with minimal side effects in women with chemotherapy refractory metastatic breast cancer. ${ }^{9}$ When bevacizumab was combined with other common therapeutic agents such as capecitibine or paclitaxel, bevacizumab associated toxicities and the development of resistance to the drug emerged. ${ }^{12}$ As a result, the FDA removed bevacizumab from the market because there was a safety concern that the risks outweighed the benefits in terms of prolonged progression free survival. ${ }^{14}$ Recently, Mackey JR et al. ${ }^{15}$ conducted a large randomized phase III trial comparing doxetaxel alone or in combination with ramucirumab, a human immunoglobulin G1 antibody that binds VEGFR2 and blocks ligand-stimulated activation. This study was a randomized double blind phase III clinical trial in patients with HER2 negative breast cancer who had not received cytotoxic chemotherapy. ${ }^{15}$ When used in combination, there was an increase in progression free survival (8.2 months to 9.5 months), but this did not reach statistical significance $(P=0.077)$ and failed to prolong overall survival. ${ }^{15}$ There were also significantly more toxicity and side effects that ranged from fatigue, hypertension, neutropenia, hand-foot syndrome, and stomatitis. ${ }^{15}$ The results of this trial were disappointing and since the FDA approved ramucirumab for gastric cancer, anti-VEGF therapy in breast cancer treatment has specifically been called into question lately due to the repeated failure of clinical results. ${ }^{46}$

A review by Sledge $\mathrm{GW}^{46}$ suggested that the failure of clinical anti-VEGF studies relies heavily on the assumption that we simply do not know who benefits from such treatment. Sledge $\mathrm{GW}^{46}$ also states that numerous mechanisms of resistance to anti-VEGF therapy have been identified including hypoxia mediated increases in tumor cell aggressiveness, recruitment of vascular progenitors, and the emergence of alternative angiogenic pathways.

Since research has still not pinpointed biological predictors of clinical success with anti-angiogenic therapies, additional work is needed to uncover novel targets for therapeutic approaches. Emerging anti-angiogenic therapies include targeting pro-angiogenic factors directly, as seen in the study by $\mathrm{He}$ et al., ${ }^{16}$ when miRNA was utilized as an inhibitor of Ang2. ${ }^{16}$ Other emerging techniques include blocking angiogenic signaling pathways such as STAT, TGF- $\beta$ and Notch. ${ }^{20,22,26,27}$ The bidirectional crosstalk between Notch receptors and EGFR suggests that angiogenic pathways are inter connected and perhaps targeting just one receptor or signal transduction pathway may not have therapeutic outcome. ${ }^{24}$ Many of these studies have in vitro effects against angiogensis, and as a result, will need to be evaluated for their efficacy in in vivo models. In addition to targeting receptors and signaling pathways, the administration of ellagic acid, a natural antioxidant found in many fruits and vegetables, has been shown to weaken the migratory potential of ECs and significantly inhibited VEGFR-2 tyrosine kinase activity through downstream signaling pathways MAPK and PI3/Akt. ${ }^{34}$ Furthermore, research has shown that proproanolol, commonly used to treat hypertension, and digoxin, commonly used to treat atrial fibrillation, significantly decreased vascular surface area, inhibited new capillary tube formation and targeted pro-angiogenic factors like HIF-1 and PDGF-B respectively. ${ }^{44,45}$ It is likely that a combined approach, wherein drugs targeting pro-angiogenic factors and their receptors in addition to pro-angiogenic signaling pathways may provide greater tumor antiangiogenic effectiveness. 


\section{Conclusion and future outlook}

While anti-angiogenic therapeutic approaches have not cured breast cancer, it is anticipated that research with these agents will prevent the expansion of tumor masses, cause tumor dormancy, or sustain regression of the tumor. The clinical utility of available antiangiogenic agent's remains uncertain due to the absence of data on overall survival, development of additional toxicities, and substantial costs associated with the ongoing research. ${ }^{15}$ The population of patients selected for research is essential since some patients with metastatic breast cancer may benefit from anti-angiogenic agents over others.

Aside from targeting pro-angiogenic factors or their downstream signaling pathways, it is foreseeable that targets may be directed against the microenvironment of the tumor vessels. In particular, future research may investigate novel mechanisms of targeting the ECM, a protein-rich entity that includes a number of proteins and proteoglycans such as collagen, the main component of connective tissue and surrounding structural support for cells and vessels, and fibronectin, a protein that also plays an essential role in wound healing. Although the overall composition varies between multi cellular structures, cellular communication, differentiation and angiogenesis are common functions of the ECM. Another potential approach may be to investigate whether vaccines have antiangiogenic therapeutic effects. Recent studies have demonstrated that vaccines show promising effects on cancer cells. Recently Zhao D et al., ${ }^{47}$ showed that live attenuated measles virus vaccine induced apoptosis and promoted tumor regression in lung cancer. Although this study only demonstrated locally induced tumor progression and not metastatic properties, its implications were promising as a therapeutic strategy for lung cancer. ${ }^{47}$ Furthermore, the use of oncolytic viruses, specifically the vaccinia virus, for the treatment of cancer is also an emerging area of cancer research and therapy that has received great attention in exerting their anti-tumor effects through genetic engineering strategies. ${ }^{48-53}$ It's foreseeable that, in addition to targeting the bulk tumor, vaccinia viruses may be utilized as an antiangiogenic therapeutic modality.

In conclusion, while there are several emerging therapeutic strategies that may prove to be beneficial for treating breast tumor angiogenesis, there is still a need for continued research to uncover alternative signaling mechanisms tumors use for sustaining angiogenesis. It's likely that a combinatorial approach in which multiple anti-angiogenic agents and/or combinations of these antiangiogenic agents with other chemotherapeutics may be more efficacious at treating breast tumors rather than single agents alone.

\section{Financial Support}

We gratefully acknowledge the PCOM Division of Research for funding of this project.

\section{Acknowledgments}

None.

\section{Conflicts of interest}

The authors have no financial conflicts of interest to declare.

\section{Funding}

None.

\section{References}

1. Goel S, Duda DG, Xu L, et al. Normalization of the vasculature for the treatment of cancer and other diseases. Physiol Rev. 2011;91(3):10711121.

2. Pandya NM, Dhalla NS, Santani DD. Angiogenesis - a new target for future therapy. Vascular Pharmacol. 2006;44(5):265-274.

3. Saaristo A, Karpanen T, Alitalo K. Mechanisms of angiogenesis and their use in the inhibition of tumor growth and metastasis. Oncogene. 2000 19(53):6122-6129.

4. Kristensen TB, Knutsson ML, Wehland M, et al. Anti-vascular endothelial growth factor therapy in breast cancer. Int $J$ Mol Sci. 2014;15(12):23024-23041.

5. Higgens M, Baselga J. Targeted therapies for breast cancer. J Clin Invest. 2011;121(10):3797-3803.

6. Folkman J, Merler E, Abernathy C, et al. Isolation of a tumor factor responsible for tumor angiogenesis. J Exp Med. 1971;133(2):275-288.

7. Jordan VC. A current view of tamoxifen for the treatment and prevention of breast cancer. British Journal of Pharmacology. 1993;110(2):507517.

8. Haran EF, Maretzek AF, Goldberg I, et al. Tamoxifen enhances cell death in implanted MCF7 breast cancer by inhibiting endothelium growth. Cancer Res. 1994;54(21):5511-5514.

9. Gagliardi A, Collins DC. Inhibition of angiogenesis by antiestrogens. Cancer Res. 1993;53(3):533-535.

10. Ferrara N, Li B, Winer J, et al. Inhibition of vascular endothelial growth factor-induced angiogenesis suppresses tumour growth in vivo. Nature. 1993;362: 841-844.

11. Cobleigh MA, Langmuir VK, Sledge GW, et al. A phase I/II doseescalation trial of bevacizumab in previously treated metastatic breast cancer. Semin Oncol. 2003;30(5 Suppl 16):117-124.

12. Miller KD, Chap LI, Holmes FA, et al. Randomized phase III trial of capecitabine compared with bevacizumab plus capecitabine in patients with previously treated metastatic breast cancer. J Clin Oncol. 2005;23(4):792-799.

13. Kathy M, Molin W, Julie G, et al. Paclitaxel plus bevacizumab versus paclitaxel alone for metastatic breast cancer. $N$ Engl $\mathrm{J} \mathrm{Med}$. 2007;357:2666-2676.

14. Montero AJ, Escobar M, Lopes G, et al. Bevacizumab in the treatment of metastatic breast cancer: Friend or foe? Curr Oncol Rep. 2012;14(1):111.

15. Mackey JR, Kerbel RS, Gelmon KA, et al. Controlling angiogenesis in breast cancer: A systematic review of anti-angiogenic trials. Cancer Treat Res. 2012;38(6):673-688.

16. He T, Qi F, Jia L, et al. MicroRNA-542-3p inhibits tumour angiogenesis by targeting angiopoietin-2. J Pathol. 2014;232(5):499-508.

17. De Palma M, Naldini L. Angiopoietin-2 TIEs up macrophages in tumor angiogenesis. Clinical Cancer Research. 2011;17(16):5226-5232.

18. Ahmad I, Iwata T, Leung HY. Mechanisms of FGFR-mediated carcinogenesis. Biochim Biophys Acta. 2012;1823(4):850-860.

19. Bono F, De Smet F, Herbert C, et al. Inhibition of tumor angiogenesis and growth by a small-molecule multi-FGF receptor blocker with allosteric properties. Cancer Cell. 2013;23(4):477-488.

20. Lin L, Hutzen B, Zuo M, et al. Novel STAT3 phosphorylation inhibitors exhibit potent growth-suppressive activity in pancreatic and breast cancer cells. Cancer Research. 2010;70(6):2445-2454. 
21. Muraoka RS, Dumont N, Ritter CA, et al. Blockade of TGF-B inhibits mammary tumor cell viability;migration, and metastasis. J Clin Invest. 2002;109(12):1551-1559.

22. Wang XG, Meng Q, Qi FM, et al. Blocking TGF- $\beta$ inhibits breast cancer cell invasiveness via ERK/S100A4 signal. Eur Rev Med Pharmacol Sci. 2014;18(24):3844-3853.

23. Jia W, Gao XJ, Zhang ZD, et al. S100A4 silencing suppresses proliferation, angiogenesis and invasion of thyroid cancer cells through down regulation of MMP-9 and VEGF. Eur Rev Med Pharmacol Sci. 2013;17(11):1495-1508.

24. Al-Hussaini H, Subramanyam D, Reedijk M, et al. Notch signaling pathway as a therapeutic target in breast cancer. Mol Cancer Ther. 2011;10(1):9-15.

25. Funahashi Y, Shawber CJ, Vorontchikhina M, et al. Notch regulates the angiogenic response via induction of VEGFR-1. J Angiogenes Res. 2010;2(1):3.

26. Wang SC, Hung MC. HER 2 over expressing and cancer targeting. Semin Oncol. 2001;28(5 Suppl 16):115-124.

27. Baker AT, Zlobin A, Osipo C. Notch-EGFR/HER2 bidirectional crosstalk in breast cancer. Front Oncol. 2014;4:360.

28. Chi A, Yun J, Osipo C, et al. The additive effects of gamma secretase inhibitor and ionizing radiation in MDA-MD-231 breast cancer cell line. American Society of Clinical Oncology. 2008;26(15):14594.

29. Holland SJ, Pan A, Franci C, et al. R428, a selective small molecule inhibitor of axl kinase, blocks tumor spread and prolongs survival in models of metastatic breast cancer. Cancer Res. 2010;70(4):1544-1554.

30. Perez R, Lanier V, Newman G. Leptin's pro-angiogenic signature in breast cancer. Cancers. 2013;5(3):1140-1162.

31. Friedman JM, Halaas JL. Leptin and the regulation of body weight in mammals. Nature. 1998;395(6704):763-770.

32. Gu JW, Young E, Patterson SG, et al. Postmenopausal obesity promotes tumor angiogenesis and breast cancer progression in mice. Cancer Biol Ther. 2011;11(10):910-917.

33. Gonzalez RR, Cherfils S, Escobar M, et al. Leptin signaling promotes the growth of mammary tumors and increases the expression of vascular endothelial growth factor (VEGF) and its receptor type two (VEGFR2). J Biol Chem. 2006;281(36):26320-26328.

34. Wang N, Wang ZY, Mo SL, et al. Ellagic acid, a phenolic compound, exerts anti-angiogenesis effects via VEGFR-2 signaling pathway in breast cancer. Breast Cancer Res Treat. 2012;134(3):943-955.

35. Parsons MF, Foster PA, Chander SK, et al. The in vivo properties of STX243: A potent angiogenesis inhibitor in breast cancer. Br J Cancer. 2008;99(9):1433-1441.

36. Fotsis T, Zhang Y, Pepper MS, et al. The endogenous oestrogen metabolite 2-methoxy- oestradiol inhibits angiogenesis and suppresses tumour growth. Nature. 1994;368(6468):237-239.

37. Tsukamoto A, Kaneko Y, Yoshida T, et al. 2-methoxyestradiol, an endogenous metabolite of estrogen, enhances apoptosis and bgalactosidase expression in vascular endothelial cells. Biochem Biophys Res Commun. 1998;248(1):9-12.
38. Ryschich E1, Werner J, Gebhard MM, et al. Angiogenesis inhibition with TNP-470, 2-methoxyestradiol, and paclitaxel in experi- mental pancreatic carcinoma. Pancreas. 2003;26(2):166-172.

39. Lakhani NJ, Sarkar MA, Venitz J, et al. 2 Methoxyestradiol, a promising anticancer agent. Pharmacotherapy. 2003;23(2):165-172.

40. Ireson CR, Chander SK, Purohit A, et al. Pharmacokinetics and efficacy of 2-methoxyoestradiol and 2-methoxyoestradiol-bis-sulphamate in vivo in rodents. Br J Cancer. 2004;90(4):932-937.

41. Newman SP, Leese MP, Purohit A, et al. Inhibition of in vitro angiogenesis by $2-$ methoxy- and 2-ethyl-estrogen sulfamates. Int $J$ Cancer. 2004;109(4):533-540.

42. Woodhouse EC, Chuaqui RF, Liotta LA. General mechanisms of metastasis. Cancer. 1997;80(8 Suppl):1529-1537.

43. Bendrik C, Robertson J, Gauldie J, et al. Gene transfer of matrix metalloproteinase-9 induces tumor regression of breast cancer in vivo. Cancer Res. 2008;68(9):3405-3412.

44. Pasquier E, Ciccolini J, Carre M, et al. Propranolol potentiates the antiangiogenic effects and anti- tumor efficacy of chemotherapy agents: Implication in breast cancer treatment. Oncotarget. 2011;2(10):797-809.

45. Schito L, Rey S, Tafani M, et al. Hypoxia-inducible factor 1dependent expression of platelet-derived growth factor B promotes lymphatic metastasis of hypoxic breast cancer cells. Proc Natl Acad Sci U S A. 2012;109(40):E2707-E2716.

46. Sledge GW. Anti-vascular endothelial growth factor therapy in breast cancer: Game over? Journal of Clinical Oncology. 2014;33(2):133-135.

47. Zhao D, Chen P, Yang H, et al. Live attenuated measles virus vaccine induces apoptosis and promotes tumor regression in lung cancer. Oncology Rep. 2013;29(1):199-204.

48. Jefferson A, Cadet V, Hielscher A. The mechanisms of genetically modified vaccinia viruses for the treatment of cancer. Critical Reviews in Oncology Hematology. 2015.

49. John R Mackey, Manuel Ramos-Vazquez, Oleg Lipatov, et al. Primary results of ROSE/TRIO-12, a randomized, placebo-controlled phase III trial evaluating the addition of ramucirumab to first line docetaxel chemotherapy in metastatic breast cancer. Journal of Clinical Oncology. 2015;33:41-148.

50. Folkman J. Antiangiogenesis in cancer therapy-endostatin and its mechanisms of action. Exp Cell Res. 2006;312(5):594-607.

51. Patel NS, Dobbie MS, Rochester M, et al. Up-regulation of endothelial delta-like 4 expression correlates with vessel maturation in bladder cancer. Clin Cancer Res. 2006;12(16):4836-4844.

52. Noguera-Troise I, Daly C, Papadopoulos NJ, et al. Blockade of D114 inhibits tumour growth by promoting non-productive angiogenesis. Nature. 2006;444(7122):1032-1037.

53. Tanaka NG, Sakamoto N, Korenaga H, et al. The combination of a bacterial polysaccharide and tamoxifen inhibits angiogenesis and tumor growth. International Journal of Radiation Oncology. 1991;60(1-2):7983. 